\title{
Diferencias de atribución causal en el bullying entre sus protagonistas
}

\author{
José María Avilés Martínez
}

Profesor de Psicología y Pedagogía en el Departamento de Orientación del IES Santa Teresa de Jesús de Valladolid

España

bullying@telefonica.net 


\section{Resumen}

Introducción. Pretendemos indagar las diferencias de atribución causal entre los participantes del bullying, según la situación de participación en el maltrato desde la que juzgan los hechos y el perfil habitual que ocupan en las situaciones de intimidación.

Método. A través de una muestra de alumnado de 10 a 18 años y a partir del cuestionario CIMEI (Avilés, 1999) se sitúa a los sujetos en los subgrupos de análisis: agresores/as, víctimas, agresores/as agredidos/as y testigos.

Resultados. Encontramos diferencias de atribución dependiendo de la situación de participación, el perfil de los sujetos y, en algunas atribuciones, en función del sexo. La consistencia explicativa en los perfiles se muestra en la tendencia de los agresores/as a la minimización y el uso de la provocación para justificar el bullying; la idea de asimetría en las víctimas y su desconocimiento sobre el por qué de lo que les pasa; la reactividad en los agresores/as agredidos/as y la identificación de la intencionalidad amoral de quienes agreden por parte de los testigos.

Conclusiones. Aspectos como la competencia y cognición sociales, las diferencias de valoración, manejo emocional y motivación y las relaciones de poder y su ejercicio en el grupo de iguales, se han identificado interactivamente importantes en la atribución que hacen los sujetos en el bullying, lo que orienta la intervención hacia el trabajo de la empatía, la interiorización de mecanismos para la prevención de los conflictos, el entrenamiento en autocontrol emocional, el aprendizaje individual y grupal en tareas de ayuda y protección de los otros y hacia la prevención del maltrato a través de la común educación moral.

Palabras clave: atribución causal, bullying, agresor, agresor-agredido, víctima, espectadores. 


\section{Introducción}

Diversas investigaciones han puesto de relieve la diversidad de causas que están detrás de la dinámica bully-victim y de factores que actuarían como protección/riesgo (Farrington, 1993; Morin y Janosz, 2006; Olweus, 1998; Ortega y Mora-Merchán, 2000; Rigby, 2002; Smith, 2005) en diversos ámbitos como el cultural, social, escolar, grupal, familiar y personal. Sin embargo, también se han indagado los argumentos de los sujetos implicados en el maltrato, su intención en los hechos y los sentimientos que les producen, así como la cantidad, signo y calidad de las herramientas que ponen en juego (Besag, 1989; Dodge, 1991; Smith et al., 1993; Sutton et al. 1999).

Por otra parte, se ha visto de gran importancia la necesidad de valorar el significado que los sujetos participantes en el bullying dan a esos hechos; el grado de ambigüedad con que los perciben y su papel en la decantación de la atribución (Smorti, 1999); cómo los comprenden e integran las emociones que les provocan (Menesini, 1999), que pueden jugar un papel importante en la guía y ponderación de sus actuaciones (Del Barrio et al., 2003; Smorti y Ciucci, 2000). Además, también se ha puesto de relieve la significación de tener presentes las diferencias entre los perfiles de los sujetos que participan en el bullying para orientar las posibles intervenciones (Toblin et al., 2005).

Estarían en juego aspectos cognitivos en la comprensión de los fenómenos grupales del maltrato; valoración de los hechos en función de la situación-posición puntual desde la que participan en el maltrato, su grado de independencia y autonomía moral; y, finalmente, de la influencia que la propia historia socioemocional de experiencias de maltrato y el perfil que el sujeto termine ocupando en la dinámica grupal, puedan aportarle en la construcción de una explicación propia sobre por qué suceden.

\section{Objetivos}

La presente investigación pretende valorar las diferencias de percepción del maltrato entre iguales (bullying) a partir de la atribución causal que hacen del mismo, según la situación de participación (ejercicio-recepción-contemplación), el perfil habitual que ocupan en 
ese escenario: agresor/a (a), víctima (b), agresor-agredido (c), o testigo del maltrato (d), y la variable sexo (Avilés, 2006).

\section{Método}

\section{Participantes}

La muestra inicial la componen 2181 sujetos de 10 a 18 años (79 del tercer ciclo de Educación Primaria y 989 del primer ciclo, y 1001 del segundo ciclo de Educación Secundaria Obligatoria) de veinte centros educativos. Los centros participantes son en su mayoría públicos (sólo uno privado) y de población urbana (sólo cinco centros son rurales) de las provincias de Álava, Valladolid y Vizcaya. La selección de los sujetos estuvo condicionada por la de los centros participantes en un proyecto de formación sobre bullying. Las aulas seleccionadas para la exploración se consideraron como naturalmente habían sido construidas en el centro y se pidió que la participación en la investigación de un aula por cada uno de los niveles que tenía cada centro. En la selección no hubo criterios de conflictividad o de capacitación intelectual. Se utilizó un protocolo de recogida de información suficientemente controlado, informado y uniforme con instrucciones comunes en el previo, durante el pase de la prueba y en la recogida y procesamiento de los datos.

De la muestra inicial se trabajó, para el análisis estadístico, con una muestra más reducida: los sujetos que encajaban en cada perfil de análisis conforme a las respuestas emitidas en el instrumento de evaluación empleado: víctimas, agresores, agresores agredidos y espectadores conforme aparece en la Tabla $n^{0} 1$.

\begin{tabular}{|c|c|c|c|}
\hline Perfil & Chicos $(51,4 \%)$ & Chicas $(48,6 \%)$ & Total $(100 \%)$ \\
\hline Agresores/as & $99(13,4 \%)$ & $27(3,9 \%)$ & $126(8,8 \%)$ \\
\hline Víctimas & $62(8,4 \%)$ & $54(7,8 \%)$ & $116(8,1 \%)$ \\
\hline $\begin{array}{l}\text { Agresores/as } \\
\text { agredidos/as }\end{array}$ & $12(1,6 \%)$ & $5(0,7 \%)$ & $17(1,2 \%)$ \\
\hline Testigos & $564(76,5 \%)$ & $610(87,6 \%)$ & $1174(81,9 \%)$ \\
\hline Total & $737(100 \%)$ & $696(100 \%)$ & $1433(100 \%)$ \\
\hline
\end{tabular}




\section{Instrumento}

El instrumento de evaluación empleado para medir las situaciones de bullying ha sido el CIMEI (Avilés, 2003) Cuestionario sobre Intimidación y Maltrato Entre Iguales. Instrumento que fue validado con la lectura de la tesis del autor (Avilés, 2002). En su versión de 2003, es un autoinforme que de deriva del CIMEI inicial (Avilés, 1999) que a su vez se construyó utilizando algunos ítems de otros cuestionarios ${ }^{1} \mathrm{e}$ incorporando ítems nuevos. El CIMEI (Avilés, 2003) se compone de 36 items de opción múltiple.

Para la consideración de los sujetos en los perfiles se procedió seleccionando como víctimas a aquellos que el item $\mathrm{n}^{\circ} 11$ respondían "Bastantes veces" y "Casi todos los días, casi siempre" a la pregunta sobre recurrencia de la victimización; como agresores/as a quienes en el item 22 cuantificaban las agresiones que realizaron en el último trimestre "Entre cinco y diez veces", "Entre diez y veinte veces" y "Más de veinte veces"; como agresores/as agredidos/as a quienes en las dos categorías anteriores (víctimas y agresores) participaban en esa intensidad y duración; y como espectadores/as a quienes en el penúltimo item del CIMEI se percibían como "Preferentemente espectador/a".

\section{Análisis Estadístico}

La atribución que hacen los sujetos del maltrato, así como sus diferencias, las hemos valorado desde las tres situaciones de participación en la dinámica del maltrato: ejercicio, padecimiento y contemplación, ejercidas en un momento determinado ${ }^{2}$. En los tres casos valoramos las diferencias comunes de atribución causal que hacen los cuatro perfiles de participación habitual: víctimas, agresores/as, agresores/as agredidos/as y testigos, es decir, los subgrupos de análisis.

Se ha utilizado el programa SPSS para todo el procesamiento estadístico de los datos con análisis de contingencia entre variables. Como el objetivo era valorar las diferencias en la percepción de atribución causal de los subgrupos se procedió a un análisis multivariente de la varianza (MANOVA) para determinar las diferencias entre los perfiles en el conjunto de variables. El resultado fue una Lambda de Wilks de .605, $F(11,182)=66, p<.001$. También se aseguró la no influencia de la distribución por sexos, cuyos análisis por separado coincidieron

\footnotetext{
${ }^{1}$ Concretamente de los cuestionarios elaborados por Ortega, Mora y Mora-Merchán (1995) y por Fernández y Ortega (Fernández García, 1998), que son a su vez reflejo del cuestionario de Olweus (1983).

${ }^{2}$ Todos los sujetos han podido estar en cada una de las situaciones y tienen información desde ellas.
} 
con los realizados al conjunto. Además se procedió a concretar las diferencias de atribución causal entre los perfiles (subgrupos) analizando la varianza de un factor (ANOVA) en cada una de las variables dependientes y entre los subgrupos desde cada situación de participación del maltrato (intimidación, victimización y contemplación) como se recoge $\mathrm{e}^{3}$ en las Tablas $\mathrm{n}^{\mathrm{o}}$ 2, 3 y 4 obteniéndose diferencias significativas en veinte de las veintidós variables dependientes.

\section{Resultados}

Los resultados muestran diferencias significativas en la atribución dependiendo de la situación de participación en la que se esté y del perfil que los sujetos habitualmente ocupen en la dinámica bully-victim, así como de género en determinadas atribuciones. Quienes son maltratados dan fuerza distinta a las razones de ese maltrato, según la posición que ocupan habitualmente en el bullying (ver Tabla 2).

Para conocer esas causas el cuestionario pregunta a los sujetos "Si te han intimidado en alguna ocasión ¿Por qué crees que lo hicieron?”

Tabla 2 Atribución causal de quienes son maltratados

\begin{tabular}{|c|c|c|c|c|c|}
\hline \multicolumn{7}{|c|}{ ATRIBUCIÓN CAUSAL DECLARADA CUANDO SON VICTIMIZADOS } \\
QUIENES HABITUALMENTE SON . \\
\hline
\end{tabular}

Para conocer las causas de la intimidación la pregunta es "Si has participado en situaciones de intimidación hacia tus compañeros/as ¿por qué lo hiciste?”

\footnotetext{
${ }^{3}$ Las diferencias significativas entre subgrupos se indican con letras en subíndice de la siguiente for- 
Tabla 3. Atribución causal de quienes intimidan

\begin{tabular}{|c|c|c|c|c|c|}
\hline \multicolumn{6}{|c|}{$\begin{array}{c}\text { ATRIBUCIÓN CAUSAL DECLARADA CUANDO INTIMIDAN } \\
\text { QUIENES HABITUALMENTE SON... }\end{array}$} \\
\hline & $(F)$ & $\begin{array}{l}\text { Agresores } \\
\qquad \text { (a) }\end{array}$ & $\begin{array}{l}\text { Víctimas } \\
\text { (b) }\end{array}$ & $\begin{array}{c}\text { Agresores } \\
\text { agredidos } \\
\text { (c) }\end{array}$ & $\begin{array}{l}\text { Espectadores } \\
\text { (d) }\end{array}$ \\
\hline No he intimidado a nadie $* * *$ & 40.262 & $6.4_{b d}$ & $42.6_{a d}$ & $11.8_{d}$ & $65.4_{a b c}$ \\
\hline Porque me provocaron*** & 22.081 & $64_{b d}$ & $38.3_{a}$ & 35.3 & $30.1_{a}$ \\
\hline Porque a mí me lo hacen otros/as*** & 28.366 & $12.8_{c d}$ & $12.2_{c d}$ & $35.3_{a b d}$ & $2.6_{a b c}$ \\
\hline $\begin{array}{l}\text { Porque son diferentes (gitanos, discapacitados, } \\
\text { extranjeros, payos, de otros sitios) } \text { ( }^{* *}\end{array}$ & 7.311 & $4_{d}$ & $3.5_{d}$ & 5.9 & $0.5_{a b}$ \\
\hline Porque eran más débiles $* * *$ & 10.076 & $4.8_{d}$ & $2.6_{c}$ & $11.8_{b d}$ & $0.7_{a c}$ \\
\hline Por molestar*** & 14.890 & $15.2_{d}$ & 7.8 & 17.6 & $3.2_{a}$ \\
\hline Por gastar una broma*** & 15.318 & $33.6_{b d}$ & $12.2_{a}$ & 29.4 & $12.4_{a}$ \\
\hline Otros & 8.232 & $10.4_{d}$ & 7.9 & $17.6_{d}$ & $3.3_{a c}$ \\
\hline
\end{tabular}

$* * *(p \leq 0.001), * *(p \leq 0.01) \mathrm{y} *(p \leq 0.05)$

Para conocer las causas que atribuyen los testigos, se les pregunta "¿Por qué crees que algunos/as chicos/as intimidan a otros/as?"

Tabla 4. Atribución causal de quienes contemplan el maltrato

\begin{tabular}{|l|c|c|c|c|c|}
\hline \multicolumn{7}{|c|}{ QTRIBUCIÓN CAUSAL DECLARADA CUANDO SON TESTIGOS DEL MALTRATO } \\
& $(F)$ & $\begin{array}{c}\text { Agresores } \\
(a)\end{array}$ & $\begin{array}{c}\text { Víctimas } \\
(b)\end{array}$ & $\begin{array}{c}\text { Agresores agredidos } \\
(c)\end{array}$ & $\begin{array}{c}\text { Espectadores } \\
(d)\end{array}$ \\
\hline Por molestar*** & 12,666 & $39.5_{d}$ & $48.2_{d}$ & $23.5_{d}$ & $61.7_{a b c}$ \\
\hline Porque se meten con ellos** & 5,751 & $34_{d} .7_{d}$ & 31.6 & 35.3 & $21.3_{a}$ \\
\hline Porque son más fuertes* & 4,887 & $24_{b}$ & $41.2_{a d}$ & 35.3 & $30.6_{b}$ \\
\hline Por gastar una broma*** & 4,091 & $39.5_{b d}$ & $21.9_{a}$ & 23.5 & $25.1_{a}$ \\
\hline Otras razones** & 2,75 & $13_{b} .7_{b}$ & $31.3_{a}$ & 29.4 & 21 \\
\hline
\end{tabular}

$* * *(p \leq 0.001), * *(p \leq 0.01) \mathrm{y} *(p \leq 0.05)$

Según el perfil que habitualmente se ocupa en la dinámica bullying hay marcadas diferencias de atribución en las distintas posiciones desde las que se participa en el maltrato. En la Tabla 5 puede apreciarse la constante más significativa en cada perfil y situación:

ma a: agresores/as, $b$ : víctimas, $c$ : agresores/as agredidos/as y $d$ : testigos. 
Tabla 5. Atribución causal predominante según perfil y posición de participación en el bullying

\begin{tabular}{|c|c|c|c|c|}
\hline \multirow{2}{*}{$\begin{array}{c}\text { POSICIÓN } \\
\text { EVENTUAL DE } \\
\text { PARTICIPACIÓN }\end{array}$} & \multicolumn{4}{|c|}{ PERFIL HABITUAL EN LA DINÁMICA BULLY-VICTIM } \\
\hline & Agresor/a habitual & Víctima habitual & $\begin{array}{l}\text { Agresor/a agre- } \\
\text { dido/a habitual }\end{array}$ & $\begin{array}{c}\text { Testigo habitual del } \\
\text { maltrato }\end{array}$ \\
\hline $\begin{array}{l}\text { Cuando es maltra- } \\
\text { tado/a }\end{array}$ & $\begin{array}{l}\text { Minimización } * b * * d \\
\text { Provocación }{ }^{* * *} b d\end{array}$ & $\begin{array}{l}\text { Desconocimiento } * * * d \\
\text { Asimetrías } * * * a d \\
\text { Intencionalidad } * * * a d\end{array}$ & $\begin{array}{c}\text { Provocación } * * * d \\
\text { Otras razo- } \\
\text { nes } * * * a d\end{array}$ & $\begin{array}{c}\text { No ser } \\
\text { víctimas } * * * a b c\end{array}$ \\
\hline Cuando maltrata & $\begin{array}{c}\text { Provocación } * * * b d \\
\text { Minimización } * * * b d \\
\text { Intencionalidad } * * a * * * d\end{array}$ & & $\begin{array}{l}\text { A mí me lo } \\
\text { hacen otros } \\
\qquad * * * a b d \\
\text { Asimetría de } \\
\text { fuerza***bd }\end{array}$ & $\begin{array}{c}\text { No ser } \\
\text { agresores/as } * * * a b c\end{array}$ \\
\hline $\begin{array}{c}\text { Cuando contempla } \\
\text { el maltrato }\end{array}$ & $\begin{array}{l}\text { Minimización } b^{* *} d^{* * *} \\
\text { Provocación } * * * \mathrm{~d}\end{array}$ & $\begin{array}{c}\text { Asimetría de } \\
\text { fuerza*ad }\end{array}$ & & Iintencionalidad $* * * a b c$ \\
\hline
\end{tabular}

El agresor/a habitual minimiza los hechos desde cualquiera de las posiciones que ocupe en el maltrato significativamente más que los testigos, y más que las víctimas, cuando maltrata y contempla el maltrato. Además, cuando está agrediendo justifica lo que hace por la provocación más que víctimas y testigos en esa situación. Cuando actúa maltratando expresa diferencias de atribución causal por intencionalidad con los espectadores. Con estos últimos, también se diferencia en provocación atribuida cuando es testigo del maltrato

El perfil de víctima ofrece diferencias de atribuciones con los testigos en su desconocimiento, las diferencias de poder y la intencionalidad cuando es victimizada y en el argumento del desequilibrio de poder cuando contempla el maltrato que sufren otros. Con los agresores/as, presenta diferencias en la atribución por desequilibrio de poder e intencionalidad cuando sufre el maltrato y en la tesis de la diferencia de poder cuando lo contempla.

El agresor/a agredido/a argumenta las causas del bullying cuando lo ejerce diferenciadamente al resto en haberlo sufrido antes él y en diferencias de poder; y en la provocación cuando es victimizado, comparado con los testigos.

Los testigos se muestran lógicamente más distantes que los otros perfiles en las situaciones de vitimización e intimidación y cuando lo contemplan atribuyen a la intencionalidad la causa del bullying significativamente más que los otros perfiles. 
Análisis de las atribuciones causales comunes a los perfiles en los items del CIMEI.

\section{$\underline{\text { La provocación }}$}

Atribuir a situaciones conflictivas previas la ocurrencia de episodios de bullying son argumentos comunes, que se utilizan con diferente intensidad desde cada perfil estable de maltrato y desde cada situación de participación (ver Tabla 6), recogidos en los ítems del cuestionario CIMEI, (ver Tablas 2, 3 y 4) al utilizar expresiones con elementos de provocación: "porque los provoqué", "porque a mi me lo hacen otros/as", "porque se meten con ellos/as"...

Tabla 6. Diferencias en la atribución causal de la provocación del bullying por los actos de otros

\begin{tabular}{|c|c|c|c|c|c|c|c|c|c|c|c|}
\hline \multicolumn{12}{|c|}{ Atribución causal: la provocación } \\
\hline \multirow{3}{*}{ 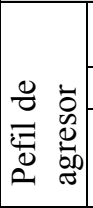 } & $\begin{array}{l}\text { Si es } \\
\text { victimizado }\end{array}$ & $15.4(* * *)(b, d)$ & \multirow{3}{*}{ 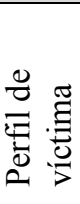 } & $\begin{array}{l}\text { Si es } \\
\text { victimizado }\end{array}$ & 6.1 & & $\begin{array}{l}\text { Si es } \\
\text { victimizado }\end{array}$ & $17.6(* *)(d)$ & \multirow{3}{*}{ 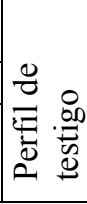 } & $\begin{array}{l}\text { Si es } \\
\text { victimizado }\end{array}$ & 2.4 \\
\hline & Si maltrata & $64(* * *)(b, d)$ & & Si maltrata & 38.8 & \multirow{2}{*}{ 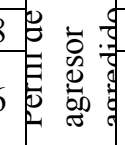 } & \multirow{2}{*}{\begin{tabular}{|l|} 
Si maltrata \\
Si contem- \\
pla el \\
maltrato
\end{tabular}} & 35.3 & & Si maltrata & 30.1 \\
\hline & $\begin{array}{l}\text { Si contem- } \\
\text { pla el } \\
\text { maltrato }\end{array}$ & $34.7(* * *)(d)$ & & $\begin{array}{l}\text { Si contem- } \\
\text { pla el } \\
\text { maltrato }\end{array}$ & 31.6 & & & 35.3 & & $\begin{array}{l}\text { Si contem- } \\
\text { pla el } \\
\text { maltrato }\end{array}$ & 21.3 \\
\hline
\end{tabular}

Entre quienes son maltratados hay diferencias significativas $(p \leq 0.01)$ a favor de los chicos como argumento explicativo del bullying. Es causa significativamente más acentuada $(p \leq 0.001)$ en los agresores/as respecto a víctimas $\mathrm{y}$ testigos, $\mathrm{y}$ en los agresores/as agredidos/as $(p \leq 0.01)$ respecto a los testigos.

Desde la posición de haber maltratado a otros, la provocación se decanta también a favor de los chicos $(p \leq 0.001)$. Es significativamente más frecuente en el agresor/a habitual $(p \leq 0.001)$ que en víctimas y testigos.

Cuando se contempla el maltrato, esa atribución la hacen significativamente más ( $p \leq$ 0.001) agresores/as que testigos. Aunque cuando se contempla el maltrato no se dan diferencias significativas de género entre perfiles, sí ocurre entre agresores habituales a favor de los chicos $(p \leq 0.001)$ y entre testigos habituales a favor de las chicas $(p \leq 0.001)$. 


\section{$\underline{\text { Las asimetrías }}$}

Las diferencias personales, de fuerza y poder entre iguales y dentro del grupo, en general, se suelen esgrimir también como razones del abuso del agresor/a sobre la víctima. Sin embargo, estas argumentaciones varían significativamente según la situación y perfil de quien las hace (ver Tablas 7a y 7b) a través de las expresiones recogidas en el CIMEI: "porque soy diferente a ellos", "porque eran más débiles", "porque son más fuertes"...

Tablas 7a y 7b. Atribuciones causales por asimetrías en perfiles y situaciones de intimidación

\begin{tabular}{|c|c|c|c|c|c|c|c|c|c|c|c|}
\hline \multicolumn{12}{|c|}{ Atribución causal: las diferencias } \\
\hline \multirow{3}{*}{ 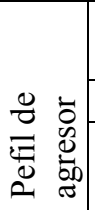 } & $\begin{array}{l}\text { Si es } \\
\text { victimizado }\end{array}$ & 8.1 & \multirow{3}{*}{ 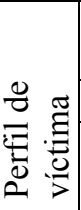 } & $\begin{array}{l}\text { Si es } \\
\text { victimizado }\end{array}$ & $30.4(* * *)(a, d)$ & & $\begin{array}{l}\text { Si es } \\
\text { victimizado }\end{array}$ & 23.5 & \multirow{3}{*}{ 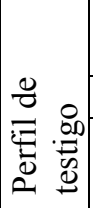 } & $\begin{array}{l}\text { Si es } \\
\text { victimizado }\end{array}$ & 3.7 \\
\hline & Si maltrata & $4(* * d)$ & & Si maltrata & $3.5(* * d)$ & \multirow{2}{*}{ 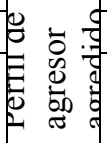 } & Si maltrata & $5.9(* d)$ & & Si maltrata & 0.5 \\
\hline & $\begin{array}{l}\text { Si contem- } \\
\text { pla el } \\
\text { maltrato }\end{array}$ & - & & $\begin{array}{l}\text { Si contem- } \\
\text { pla el } \\
\text { maltrato }\end{array}$ & - & & $\begin{array}{l}\text { Si contem- } \\
\text { pla el } \\
\text { maltrato }\end{array}$ & - & & $\begin{array}{l}\text { Si contem- } \\
\text { pla el } \\
\text { maltrato }\end{array}$ & - \\
\hline
\end{tabular}

\begin{tabular}{|c|c|c|c|c|c|c|c|c|c|c|c|}
\hline \multicolumn{12}{|c|}{ Atribución causal: la desigualdad de fuerzas } \\
\hline \multirow{3}{*}{ 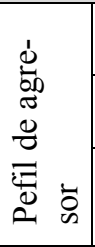 } & $\begin{array}{l}\text { Si es } \\
\text { victimizado }\end{array}$ & 12.9 & \multirow{3}{*}{ 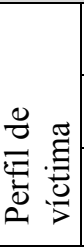 } & $\begin{array}{l}\text { Si es } \\
\text { victimizado }\end{array}$ & $36.5(* * *)(a, d)$ & \multirow{3}{*}{ 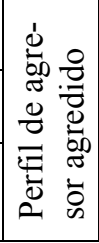 } & $\begin{array}{l}\text { Si es } \\
\text { victimizado }\end{array}$ & 23.5 & \multirow{3}{*}{ 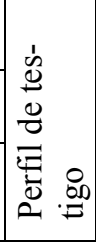 } & $\begin{array}{l}\text { Si es } \\
\text { victimizado }\end{array}$ & 4.2 \\
\hline & Si maltrata & 4.8 & & Si maltrata & 2.6 & & Si maltrata & $\begin{array}{l}11.8\left(a^{*},\right. \\
\left.b^{* *}, d^{* * *}\right)\end{array}$ & & Si maltrata & 0.7 \\
\hline & $\begin{array}{l}\text { Si contem- } \\
\text { pla el } \\
\text { maltrato }\end{array}$ & 2.4 & & $\begin{array}{l}\text { Si contem- } \\
\text { pla el } \\
\text { maltrato }\end{array}$ & $\begin{array}{l}41.2(* * * a, \\
* * d)\end{array}$ & & $\begin{array}{l}\text { Si contem- } \\
\text { pla el } \\
\text { maltrato }\end{array}$ & 35.3 & & $\begin{array}{l}\text { Si contem- } \\
\text { pla el } \\
\text { maltrato }\end{array}$ & 30.6 \\
\hline
\end{tabular}

Entre quienes son maltratados, las atribuciones asimétricas son reconocidas mucho más $(p \leq 0.001)$ por las víctimas que por el resto, especialmente que agresores/as y testigos.

Entre quienes maltratan, las razones de asimetría referidas a desequilibrio de fuerzas, las dan más los agresores/as agredidos/as que cualquier otro perfil - espectadores $(p \leq 0.001)$, víctimas $(p \leq 0.01)$ y agresores/as $(p \leq 0.05)-$. Las referidas a diferencias personales las esgrimen significativamente más cualquier perfil activo del maltrato - agresores/as $(p \leq 0.01)$, víctimas $(p \leq 0.01)$ y agresores/as agredidos/as $(p \leq 0.05)$ - que los testigos.

Si consideramos a quienes contemplan el maltrato, son las víctimas habituales las que echan mano del argumento asimétrico para justificar el bullying que observan, significativamente más que agresores/as $(p \leq 0.001)$ y que testigos $(p \leq 0.01)$.

Por otra parte, las razones de asimetría, no están entre los argumentos que separan a chicos y chicas para justificar el maltrato. Sólo son las chicas víctimas y las chicas agresoras agredidas quienes presentan diferencias significativas $(p \leq 0.001)$ en esto respecto a sus compañeros como argumento explicativo del maltrato cuando llegan a ser victimizados. 


\section{$\underline{\text { La intencionalidad }}$}

El bullying es considerado un comportamiento agresivo que pretende un daño, utilizando la planificación y procedimientos que están en la base de su intencionalidad. La intencionalidad la indican expresiones como "por molestarme" o "por molestar" (ver Tabla 8).

Cuando se sufre el maltrato, son las víctimas habituales quienes significativamente ( $p \leq$ $0.001)$ identifican este argumento más que agresores/as y testigos.

En el ejercicio del maltrato, son los agresores/as habituales quienes reconocen significativamente más que testigos $(p \leq 0.001)$ y víctimas $(p \leq 0.01)$ esta causa como motor de la intimidación. Y víctimas $(p \leq 0.05)$ y agresores/as agredidos/as $(p \leq 0.05)$ más que testigos.

Cuando se contempla el maltrato, son quienes no están implicados directamente los que significativamente $(p \leq 0.001)$ recurren a este argumento más que el resto de perfiles.

Tabla 8. Diferencias atribucionales de "por molestar"

\begin{tabular}{|c|c|c|c|c|c|c|c|c|c|c|c|}
\hline \multicolumn{12}{|c|}{ Atribución causal: la intencionalidad "por molestar" } \\
\hline \multirow{3}{*}{ 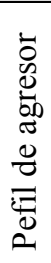 } & $\begin{array}{l}\text { Si es } \\
\text { victimi- } \\
\text { zado }\end{array}$ & 12.2 & \multirow{3}{*}{ 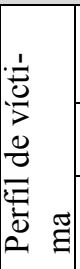 } & $\begin{array}{l}\text { Si es } \\
\text { victimi- } \\
\text { zado }\end{array}$ & $\begin{array}{l}34.8(* * * a, \\
\text { d) }\end{array}$ & \multirow{3}{*}{ 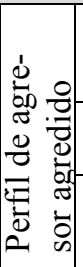 } & $\begin{array}{l}\text { Si es } \\
\text { victimi- } \\
\text { zado }\end{array}$ & 23.2 & \multirow{3}{*}{ 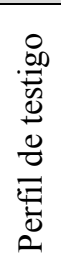 } & $\begin{array}{l}\text { Si es victi- } \\
\text { mizado }\end{array}$ & 9.7 \\
\hline & $\begin{array}{l}\text { Si maltra- } \\
\text { ta }\end{array}$ & $\begin{array}{l}15.2(* * * d * * \\
\text { b) }\end{array}$ & & $\begin{array}{l}\text { Si maltra- } \\
\text { ta }\end{array}$ & $7.8(* d)$ & & $\begin{array}{l}\text { Si maltra- } \\
\text { ta }\end{array}$ & $17.6(* d)$ & & Si maltrata & 3.2 \\
\hline & $\begin{array}{l}\text { Si con- } \\
\text { templa el } \\
\text { maltrato }\end{array}$ & 39.5 & & $\begin{array}{l}\text { Si con- } \\
\text { templa el } \\
\text { maltrato }\end{array}$ & 48.2 & & $\begin{array}{l}\text { Si con- } \\
\text { templa el } \\
\text { maltrato }\end{array}$ & 23.5 & & $\begin{array}{l}\text { Si contem- } \\
\text { pla el } \\
\text { maltrato }\end{array}$ & $\begin{array}{l}61.7\left(^{*}\right. \\
* * a, b, c)\end{array}$ \\
\hline
\end{tabular}

Por sexos, la atribución causal "por molestar" no presenta diferencias significativas entre chicos y chicas, ni cuando intimidan ni cuando son maltratados, pero sí las presenta desde la contemplación a favor de las chicas $(p \leq 0.01)$.

\section{$\underline{\text { La minimización }}$}

Presente en muchas concepciones sociales, familiares y escolares, restar importancia a los sucesos, atribuirlos a causas como la broma, las dinámicas relacionales o las contingencias de las situaciones, son exponentes de trivialización y justificación del maltrato. La expresión "por gastar una broma" refleja minimización y no arroja la misma presencia ni entre las distintas situaciones del maltrato ni entre los perfiles del mismo (ver Tabla 9). 
Cuando se sufre el maltrato, es el agresor/a habitual el que prefiere esta atribución para explicar el maltrato más que víctimas $(p \leq 0.05)$ y testigos $(p \leq 0.001)$. Además, esta tendencia se da más en los agresores habituales masculinos que en las agresoras.

Durante el ejercicio del maltrato, la minimización la usan más chicos $(p \leq 0.001)$ que chicas y el argumento respalda a agresores/as $(p \leq 0.001)$ más que a víctimas y testigos.

$\mathrm{Al}$ contemplar los hechos, siguen siendo los agresores/as habituales quienes adjudican esta causa significativamente más que victimas $(p \leq 0.01)$ y testigos $(p \leq 0.001)$.

Tabla 9. Diferencias de atribución en la minimización del maltrato

\begin{tabular}{|c|c|c|c|c|c|c|c|c|c|c|c|}
\hline \multicolumn{12}{|c|}{ Atribución causal: la minimización "por bromear" } \\
\hline \multirow{3}{*}{ 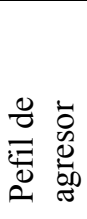 } & $\begin{array}{l}\text { Si es } \\
\text { victimizado }\end{array}$ & $20.3\left(* a^{* * *} d\right)$ & \multirow{3}{*}{ 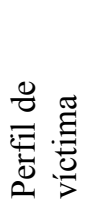 } & $\begin{array}{l}\text { Si es } \\
\text { victimizado }\end{array}$ & 9.6 & \multirow{3}{*}{ 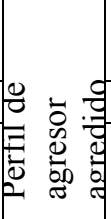 } & \multirow{3}{*}{\begin{tabular}{|l}
$\begin{array}{l}\text { Si es } \\
\text { victimizado }\end{array}$ \\
Si maltrata \\
Si contem- \\
pla el \\
maltrato
\end{tabular}} & \multirow{2}{*}{$\begin{array}{l}11.8 \\
29.4 \\
\end{array}$} & \multirow{3}{*}{ 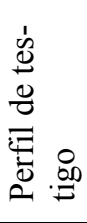 } & \multirow{2}{*}{$\begin{array}{l}\text { Si es } \\
\text { victimizado } \\
\text { Si maltrata }\end{array}$} & \multirow{2}{*}{$\begin{array}{l}9.8 \\
12.4\end{array}$} \\
\hline & Si maltrata & $33.6(* * * b d)$ & & Si maltrata & 12.2 & & & & & & \\
\hline & $\begin{array}{l}\text { Si contem- } \\
\text { pla el } \\
\text { maltrato }\end{array}$ & $39.5\left(b^{* *} d^{* * *}\right)$ & & $\begin{array}{l}\text { Si contem- } \\
\text { pla el } \\
\text { maltrato }\end{array}$ & 21.9 & & & 23.5 & & $\begin{array}{l}\text { Si contem- } \\
\text { pla el } \\
\text { maltrato }\end{array}$ & 25.1 \\
\hline
\end{tabular}

La minimización está significativamente más presente en los chicos $(p \leq 0.001)$ cuando maltratan. En la victimización, las diferencias se presentan más entre chicos agresores habituales $(p \leq 0.001)$ que en sus compañeras. Desde la contemplación no se presentan diferencias. Podemos resumir en la Tabla 10 aquellas relaciones significativas más relevantes ${ }^{4}$.

Tabla 10. Diferencias significativas de atribución causal entre los perfiles del bullying y por sexo ${ }^{5}$.

\begin{tabular}{|c|c|c|c|c|c|c|c|c|c|c|c|}
\hline & & \multicolumn{2}{|c|}{$\begin{array}{l}\text { AGRESORES } \\
\text { HABITUALES }\end{array}$} & \multicolumn{2}{|c|}{$\begin{array}{l}\text { VÍCTIMAS HABI- } \\
\text { TUALES }\end{array}$} & \multicolumn{2}{|c|}{$\begin{array}{l}\text { AGRESORES/AS } \\
\text { AGREDIDOS/AS }\end{array}$} & \multicolumn{2}{|c|}{ TESTIGOS } & \multirow[t]{2}{*}{ CHICOS } & \multirow[t]{2}{*}{ CHICAS } \\
\hline & & CHICOS & CHICAS & CHICOS & CHICAS & CHICOS & CHICAS & CHICOS & CHICAS & & \\
\hline \multirow{4}{*}{ 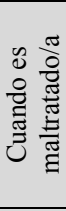 } & PROVOCACIÓN & & & & & \multicolumn{2}{|c|}{$* * *$} & & & $* *$ & \\
\hline & DIFERENCIA & & & \multicolumn{2}{|c|}{$* * *_{2}$} & & $* * *$ & & & & \\
\hline & INTENCIONALIDAD & & & \multicolumn{2}{|c|}{$* * *$} & & & & & & \\
\hline & MINIMIZACIÓN & $* * *$ & & & & & & & & & \\
\hline \multirow{4}{*}{ 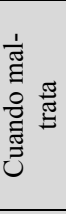 } & PROVOCACIÓN & \multicolumn{2}{|c|}{$* * *$} & & & & & & & $* * *$ & \\
\hline & DIFERENCIA & & & & & \multicolumn{2}{|c|}{$* * *$} & & & & \\
\hline & INTENCIONALIDAD & \multicolumn{2}{|c|}{$* * *$} & & & & & & & & \\
\hline & MINIMIZACIÓN & \multicolumn{2}{|c|}{$* * *_{1}$} & & & & & & & $* * *$ & \\
\hline \multirow{4}{*}{ 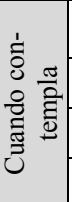 } & PROVOCACIÓN & $* * *$ & & & & & & & $* * *$ & & \\
\hline & DIFERENCIA & & & \multicolumn{2}{|c|}{$* * *$} & & & & & & \\
\hline & INTENCIONALIDAD & & & & & & & \multicolumn{2}{|c|}{$* * *_{2}$} & & \\
\hline & MINIMIZACIÓN & \multicolumn{2}{|c|}{$* * *$} & & & & & & & & \\
\hline
\end{tabular}

\footnotetext{
${ }^{4}$ Las casillas sombreadas unidas expresan diferencias significativas respecto a otros perfiles. Las sobreadas dividas expresan diferencias de sexo en el mismo perfil.
} 


\section{Discusión}

Los resultados de la investigación ponen de manifiesto contrastes revelados por otros estudios sobre diferencias con que los participantes en el bullying viven e interpretan el maltrato desde las situaciones puntuales y los perfiles habituales que ocupan en él (Olweus, 1998; Ortega, 1992; Ortega y Mora-Merchán, 2000; Whitney y Smith, 1993). Además, ahondan y profundizan en la diversidad de argumentaciones y atribuciones causales que hacen los sujetos envueltos en esas dinámicas.

Esas diferencias pueden estar en la base de una falta de acuerdo a la hora de valorar los hechos del maltrato. Así, se pone de manifiesto la necesidad llegar a acuerdos sobre lo que el alumnado percibe como bullying (Lee, 2004) y aquello que no lo es. Incluso, la necesidad de abordar ese trabajo entre otros miembros de la Comunidad Educativa (Avilés, 2005). Otras diferencias han sido explicadas desde la competencia social de los sujetos, tanto por déficit personal, error o confusión en alguno de los procesos de obtención de la información social y su interpretación (Dodge, et al., 1986), como desde la idea de dotación y habilidad (Sutton, et al., 1999). También se han dado explicaciones desde las diferencias de motivación y de instrumentalización que de las situaciones sociales hacen los sujetos, en especial los agresores/as, para la consecución de algún logro (Diaz-Aguado, 1996; Tedeschi, 1994). Incluso, ha llegado a manifestarse la idea de necesidad de obtención de dominio sobre alguien que es más débil y en el que se puede imponer el esquema dominio-sumisión (Smith y Sharp, 1994)

Un primer aspecto importante que la investigación destaca es el de que se producen atribuciones causales diferentes dependiendo del perfil habitual que el alumno/a ocupe en la dinámica bullying (Smith, et al., 1993; Toblin, et al., 2005).

Además, los datos ponen de manifiesto preferencias argumentales de atribución causal según el perfil habitual que ocupan los sujetos en la dinámica bully-victim, y consistencia explicativa de su atribución causal a lo largo de las distintas situaciones en las que se ven envueltos (Del Barrio, et al., 2003).

Así, se dan razones minimizadoras en perfiles agresores/as, especialmente en los chicos. Los agesores/as habituales tienden a explicarse lo que ocurre quitándole importancia en todas las situciones de participación, con lo que mantienen una consistencia explicativa de los hechos. Así lo explica Slee (1993) al considerarlos habituados a ese tipo de conducta agresiva o carentes de otra forma de establecer vínculos sociales (Hoover, Oliver y Hazler, 1992). Esta

\footnotetext{
${ }^{5}$ El subíndice $\left[{ }^{\star * *}{ }_{1}\right]$ indica diferencias significativas a favor de los chicos. El subíndice $\left[{ }^{\star * *}{ }_{2}\right]$ a favor de
} 
predisposición en los procesos de captación de información social (Toblin et al, 2005), les justificaría en una valoración positiva de los hechos agresivos (Olweus, 1998) y les dificultaría para valorar negativamente las agresiones, especialmente las sociales (Ortega y MoraMerchán, 2000), sobre todo si les reportan objetivos y/o carencia de respuestas opuestas (Avilés, 2002), y les diferenciaría del resto de perfiles a este respecto. Además, recurrir a razones minimizadoras para explicar el maltrato coincide con sus dificultades para reconocer las emociones en los otros (Caprara y Pastoreli, 1989) o su negativa y/o dificultad para reconocer la infelicidad en sus víctimas (Smith, et al., 1993) o hacerlo en su propia ventaja (Menesini, 1999), al ser capaces de entender mejor que ellas los estados mentales de los otros (Smorti, 1999).

Las razones justificativas del maltrato se plasman en la provocación y reacción agresiva en los perfiles agresivos, aunque no preferentemente desde una situación de participación. Exponente de la exculpación (Olweus, 1998), los agresores/as argumentan la provocación más que el resto de perfiles cuando agreden y contemplan el maltrato. Esto se explica desde modelos interpretativos de déficits en los procesos de cognición social (Crick y Dodge, 1996) y de atribuciones erróneas (Dodge, 1991) u hostiles a las relaciones con los otros (Hubbard, et al., 2001). Cuando sufren maltrato son los agresores/as agredidos/as quienes recurren a victimizaciones sufridas ( "a mí me lo hacen otros") como atribución causal más que otros perfiles, lo que explica su doble participación en el bullying, situando su acción de maltratar conectada a la de sufrir victimización. Denotan conciencia y certeza de lo que les genera su agresión y una explicación de su dinámica agresión-victimización.

Las razones intencionales que explican el maltrato se reconocen en todas las situaciones de participación con diferencias en los perfiles. Parece una razón compartida y aceptada. Algo lógico, si de lo que estamos hablando es de bullying (Besag, 1989; Olweus, 1999). Obtener algún beneficio o lograr algún objetivo (Thompson, Arora y Sharp, 2001) está en la base de la agresión instrumental (Díaz-Aguado, 1996) o proactiva (Dodge y Crick, 1990). Esta intencionalidad, reconocida por las víctimas habituales más que por otros perfiles, también la reconocen agresores/as y agresores/as agredidos/as en sus ataques, lo que refleja una conciencia de culpa que no suelen admitir socialmente (Olweus, 1998). Además, esta intencionalidad que los testigos habituales también argumentan mientras contemplan el maltrato, les coloca en posición más objetiva para construir juicios morales y valorar socialmente el daño sobre las víctimas, incluso, el disfrute de los agresores/as (Del Barrio, 1999). Esto apoya su 
comprensión moralmente adecuada del fenómeno y cierta independencia de intenciones y emociones dirigidas hacia ellos, aunque les compromete en dinámicas grupales que en pocas ocasiones saben romper (Avilés, 2006) y que ponen de relieve la diversidad de causas que los mantienen inermes ante la agresión (miedo a ser los próximos, ignorancia, dudas, no saber cómo intervenir,...).

Finalmente, las razones por asimetría de fuerzas o por diferencias que se instalan en las víctimas cuando son objeto y testigos del bullying, les hacen conscientes de sus diferencias personales y de fuerza en el grupo como explicación de lo que les pasa. Esa conciencia de desequilibrio y diferencias apoya la idea de acumulación de responsabilidad por lo que les pasa (Graham y Juvonen, 1998) con los agresores/as, aceptación de incapacidad para reequilibrarlo, y en algunos casos, de culpabilización.

\section{Implicaciones de intervención}

Si nos centramos en los cuatro ámbitos de atribución causal analizados podemos señalar algunas contrapartidas de intervención educativa en cada uno de ellos.

Abordar la minimización del matrato con los agresores/as supone hacer un trabajo de valoración de los hechos con ellos mismos y con el grupo. Para ello, parece necesario que puedan argumentar en climas de objetividad, evitando culpabilizaciones previas. En este sentido, adoptar un modelo de intervención (Avilés, 2006) idóneo puede ayudar a ello. Además exige haber realizado previamente una definición del maltrato (Lee, 2004) con la generalidad del alumnado (acotar y delimitar qué es bullying, qué no lo es y qué puede llegar a serlo). Actuaciones dirigidas a acceder al conocimiento de los sentimientos de las víctimas (Sullivan, 2001) y, por tanto, a trabajar con ellos/as la empatía, puede arrojar a los agresores/as luz para valorar de otra forma los hechos. Su tratamiento a través de la dramatización de situaciones y del roleplay, el visionado de videos o el uso de narraciones, permite valorar los hechos en frío y en sí mismos como eso, hechos con un determinado signo moral, independientemente de la individualidad de sus destinatarios (recepción, integración, gestión y resistencia de las víctimas). Ello puede ayudar también a tomar conciencia de los efectos de las propias acciones sobre los otros y sobre el grupo, permite cierta separación entre la valoración que se hace de los agresores/as y la de sus acciones (Maines y Robinson, 2003), facilitando cierta reconducción de situaciones, otras lecturas de los hechos y segundas oportunidades. Por otra parte, se muestran relevantes dos cuestiones derivadas de la participación en este trabajo de los sujetos 
que no están directamente implicados en las situaciones, los testigos. La socialización y soporte grupal del derecho a no ser maltratado por nadie, que rompa la "cultura del chivato" y salvaguarde así la indefensión individual y grupal ante el abuso; y la manifiestación social del valor y sentido que los testigos dan -intencionalidad (ver Tabla 8)- a las acciones de los agresores sobre las víctimas, con una toma de posición moral activa (verbalización social del rechazo a quienes agreden) y adopción de acuerdos grupales (normas grupales antibullying).

El trabajo sobre las atribuciones reactivas y de provocación exige una tarea preventiva general en procesos de convivencia social y escolar, que haga cotidianos instrumentos para la resolución de los conflictos (Torrego, 2000) o mecanismos mediadores (Ortega y del Rey, 2003) para el entendimiento interpersonal, y equilibrados los modelos disciplinarios que utiliza la Comunidad Educativa y sus miembros para regular la micro y macroconvivencia. Grupalmente se hacen necesarias tareas de definición y valoración del maltrato (Lee, 2004) y de aspectos normativo-convivenciales del grupo (normas antibullying). Individualmente, con los agresores/as es necesario abordar estrategias de reestructuración cognitiva e integración emocional de sus experiencias sociales a través del análisis narrativo (Del Barrio et al., 2003) y programas de autocontrol y control de la ira para los casos de errores atribucionales y reactividad agresiva. Con los agresores/as agredidos/as, su comportamiento disruptivo, desregulado conductualmente y contradictorio exige plantear acciones de doble dirección: autorregulación conductual, y, ayuda y protección.

El conocimiento compartido más o menos explícitamente por todos/as sobre la intencionalidad del bullying, favorece acciones institucionales y contextuales, de rechazo y ayuda (campañas, foros, celebraciones, lemas, declaraciones,...) en la cultura escolar; grupales, de análisis, toma de conciencia moral y adopción de acuerdos comunes (análisis de casos, normas antibullying,...); e individuales, de protagonismo de los espectadores/as del bullying que refuercen su juicio moral sobre los hechos, promocionen la conciencia de idenfensión grupal ante el maltrato en el grupo y favorezcan su implicación social en estructuras de apoyo a sus compañeros/as, como las de alumnado ayudante (Fernández et al., 2002), círculo de amigos (Sullivan, 2001) o alumnado consejero (Cowie y Sharp, 1996).

Abordar el trabajo sobre las atribuciones asimétricas exige contar con todos/as, pero especialmente con los espectadores/as y las víctimas. Es necesario romper la cultura grupal del "chivato" confrontando la valiente idea de defensa de los derechos humanos y del más débil, a la cobardía de ejercer el abuso sobre quien es menos fuerte y la intolerancia sobre el diferente. Con las víctimas es necesaria una tarea social equibradora de sus déficits desde las 
figuras de autoridad escolar y desde un trabajo individual dirigido en autoafirmación y aserción (Morganet, 1995); y con los iguales, de acompañamiento-apoyo mediante redes de ayuda, protección y consejo (Thompson, et al., 2002).

Otras atribuciones causales no analizadas explícitamente merecen también alguna indicación. El desconocimiento que atribuyen las víctimas diferenciadamente sobre lo que les pasa debe ser tratado con exiquisita confidencialidad y recíproca coordinación por parte de los adultos en la variación de las condiciones en que se produjera el bullying; compensado con tareas de concienciación y de apoyo-acompañamiento continuado de iguales y adultos, así como de mensajes y signos palpables de compromiso institucional de la Comunidad Escolar. La distancia de los espectadores/as de las experiencias de víctimas y agresores/as en el maltrato exige implicarles proporcionándoles conocimiento a través de su participación en las actividades de inserción curricular y difusión de actividades que programen los docentes contra el maltrato; dándoles protagonismo en las actuaciones organizativas del centro y en las estructuras de apoyo que se generen en el grupo; y visualizándolos en los compromisos institucionales de la Comunidad Educativa de lucha contra el maltrato.

\section{Conclusiones}

Los resultados de esta investigación ponen de manifiesto las diferencias de atribución de los participantes en el bullying, cuyo conocimiento debe permitir intervenir más centrada y efectivamente. Para conseguirlo es preciso brindar espacios donde los protagonistas puedan compartir sus opiniones, creencias, sentimientos y emociones -las explicaciones de los agresores, los sentimientos de las víctimas y el compromiso de los testigos en la resolución-; es preciso que tomen conciencia de las situaciones de maltrato, las acoten, identifiquen y definan, desvelando los prejuicios y las actitudes contradictorias de quienes se ven implicados/as. La consistencia explicativa en las atribuciones de los perfiles que refleja la investigación exige programas estables, globales y compartidos, donde puedan integrarse las actuaciones comunes con las individualizadas y fragüen actuaciones en la Comunidad Educativa. Ello es clave para que una intervención sea coherente sobre los contextos escolares, grupales, en el microcontexto del maltrato y sobre la percepción que los sujetos acumulan de sus experiencias sociafectivas con el grupo y las emociones tenidas en esas situaciones. Futuras investigaciones deberán explorar la eficacia de estas intervenciones en los cambios atribucionales de los sujetos. 


\section{Referencias}

Avilés, J.M. (1999). CIMEI. Cuestionario sobre intimidación y maltrato entre iguales. Valladolid: Autor.

Avilés, J. M. (2002). La intimidación entre iguales (bullying) en la Educación Secundaria Obligatoria. Validación del Cuestionario CIMEI y estudio de incidencia. Tesis doctoral no publicada. Universidad de Valladolid.

Avilés, J.M. (2003). CIMEI. Cuestionario sobre intimidación y maltrato entre iguales. Cuestionario no publicado.

Avilés, J.M. (2005). Intervenir contra el bullying en la Comunidad Educativa. www.concejoeducativo.org $(8$ diciembre 2005).

Avilés, J.M. (2006). Bullying: el maltrato entre iguales. Agresores, víctimas y testigos en la escuela. Salamanca: Amarú.

Barrio, C. del (1999). The use ofsemistructured interviews and qualitative methods for the study of peer bullying. Http://www.gold.ac.uk/tmr/reports/aim2 madrid1.html(25 abril 2005).

Barrio, C. del, Martín, E., Almeida, A. y Barrios A. (2003). Del maltrato y otros conceptos relacionados con la agresión entre escolares. Infancia y Aprendizaje, 26(1), pp.9-24

Besag, V. (1989). Bullies and Victims in School. Open University Press. Milton Keines.

Caprara, G.V. y Pastorelli, C. (1989). Toward a reorientation of research on agrression. European journal of personality, 3,121-138.

Cowie, H. y Sharp, S. (1996). Peer counseling schools. London: David Fulton Publishers.

Crick, N.R. y Dodge, K.A. (1996). Social information-processing mechanisms in reactive and proactive aggression. Child Development, 67, 993-1002.

Díaz-Aguado, M. J. (1996). Programas de educación para la tolerancia y prevención de la violencia en los jóvenes. Madrid: Ministerio de trabajo y asuntos sociales.

Dodge, K. (1991). The structure and function of reactive and proactive aggression. En D. Pepler y K. Rubin (Eds.), The development and treatment of childhood aggression, 201-218. Hillsdale, NJ: Erlbaum.

Dodge, K.A., Pettit, G.S., McClaskey, C.L. y Brown, M.M. (1986). Social competence in children. Monographs of the society for research in Child Development, 51 (2), 1-85.

Dodge, K. y Crick, N. R. (1990). Social information processing bases of aggressive behavior in children. Personalitiy and social psychology bulletin, 53,1.146-158. 
Farrington, D.P. (1993). Understanding and preventing bullying. Crime and justice. Review of Research, 17. Chicago: The university of Chicago press.

Fernández, I. (1998). Prevención de la violencia y resolución de conflictos. El clima escolar como factor de calidad. Madrid: Narcea Ediciones.

Fernández, I., Villaoslada, E. y Funes, S. (2002). Conflicto en el centro escolar. Madrid: Catarata.

Graham, S. y Juvonen, J. (1998). Self-blame and peer victimization in middle school: an attributional analysis. Developmental psychology, 34,3,587-599.

Hoover, J. H., Oliver, R. y Hazler, R.J. (1992). Bullying: perceptions of adolescent victims in the Midwestern USA. School Psychology International, 13, 5-16.

Hubbard, J. A., Dodge, K. A., Cillessen, A. H. N., Coie, J. D., y Schwartz, D. (2001). The dyadic nature of social information processing in boys'reactive and proactive aggression. Journal of personality and social psychology, 80,268-280.

Lee, C. (2004). Preventing bullying in schools. London: Paul Chapman Publishing.

Maines, B. Y Robinson, G. (2003). All for Alex. A Circle of Friends. Bristol: Lucky Duck Publishing.

Menesini, E. (1999). Bullying and emotions. En http://www.gold.ac.uk/tmr/reports/aim2 firenze2.html (10 febrero 2005).

Morin, A. y Janosz, M. (2006). Violence en milieu scolaire, troubles intériorisés et facterus de risques individuals et familiaux: mediation ou modération. En 3rd Internacional conference on violence in schools, pp.33-34, Bordeaux.

Morganett, R. S. (1995). Técnicas de intervención psicológica para adolescentes. Barcelona: Martínez Roca.

Olweus, D. (1993). Bullying at school. What we know and what we can do. Oxford: Blackwell.

Olweus, D. (1998). Conductas de acoso y amenaza entre escolares. Madrid: Morata.

Olweus, D. (1999). Sweden. En P.K. Smith, Y. Morita, J. Junger-Tas, D. Olweus, R. Catalano y P. Slee (Eds.), The nature of school bullying, (pp.7-27). London: Routlegde.

Ortega, R. (1992). Violence in Schools. Bully-victims Problems in Spain, Vth. European Conference on Developmental Psychology, (pp.27). Sevilla.

Ortega, R. y Mora-Merchán, J. A. (1995). Víctimas y agresores. La autopercepción del maltrato entre compañeros escolares en el paso de la enseñanza primaria a la secundaria. Comunicación presentada en el II Congreso internacional de Psicología y Educación: Intervención psicopedagógica. Madrid. Pág. 143 del libro de actas. 
Ortega, R. y Mora-Merchán, J. A. (2000). Violencia escolar: Mito o Realidad. Sevilla: Mergablum.

Ortega, R. y Rey, R. del (2003). La violencia escolar. Estrategias de prevención. Barcelona: Graó.

Rigby, K. (2002). New perspectives on bullying. London: JK Publishers.

Slee, P. T. (1993). Bullying: a preliminary investigation of its nature and effects of social cognition. Early child development and care 87,47-57.

Smith, P. K. (2005). Violencia escolar y acoso: factores de reisgo familiares. En J. Sanmartín (Coord.) Violencia y escuela. (59-76). Valencia: Centro Reina Sofía.

Smith, P.K., Bowers, L., Binney, V. y Cowie, H. (1993). Relationship of children involved in bully-victim problems at school. En S. Duck (ed.), Understanding relationship process, Vol.2: Learning about relationships. Newbury Park, CA.: Sage Publicantions.

Smith, P.K. y Sharp, S. (1994). School bullying. Insights and perspectives. Routeledge. London.

Smorti, A. (1999). Theory of mind in bullying: a methodological reassessment. En http://www.gold.ac.uk/tmr/reports/aim2 firenze1.html.(22 septiembre 2005).

Smorti, A. y Ciucci, E. (2000). Narrative strategies in bullies and victims. En Aggressive Behaviour26,33-48.

Sullivan, K. (2001). The anti-bullying handbook. Singapore: Oxford University Press.

Sutton, J., Smith, P.K. y Swettenham, J. (1999). Bullying and "theory of mind": a critique of the “social skills deficit" view of anti-social behaviour. Social developmental 8,117-127.

Tedeschi, J. T. (1984). A social psychological interpretation of human aggression. En A. Mummendey (ed), Social psychology of aggression. Heidelberg: Springer-Verlag.

Thompson D., Arora T. y Sharp S. (2002). Bullying. Effective strategies for long-term improvement. London: Routledge/Falmer.

Toblin, R. L., Schwartz, D., Hopmeyer, A., Abou-ezzeddine, T. (2005). Social-cognitive and behavioral attributes of aggressive victims of bullying. Applied developmental psychology 26,329-346.

Torrego, J. C. (2000). Modelos de regulación de la convivencia. Cuadernos de pedagogía, 304, $22-28$.

Whitney, I. y Smith, P.K. (1993). A survey of the nature and extent of bullying in junior/-middle and secondary. Educational Research, 35, 3-25. 